\title{
Change Detection on SAR data using PCA Algorithm
}

\author{
${ }^{1}$ Vijay Kumar, ${ }^{2}$ Rahul Gupta, ${ }^{1}$ Supreet Kaur, ${ }^{1}$ Saurabh Srivastava \\ Computer Science and Engineering \\ ${ }^{1}$ Lovely Professional University, ${ }^{2}$ Kanpur Institute of Technology \\ iam100rabh@gmail.com
}

\begin{abstract}
In this paper, we proposed an approach for unsupervised change detection technique on SAR data. Change detection is process of automatically identifying and analyzing the regions which undergone some changes such as spatial or spectral changes. As various techniques are available to change detection but are used for general satellite images. In order to detect change on SAR images we use PCA technique which detect the change by finding the different pixel using threshold level and compare with earlier image one.
\end{abstract}

Keywords: - PCA (Principle Component Analysis), multispectral SAR (Synthetic Aperture Radar) images, Change Detection.

\section{INTRODUCTION}

Change detection plays an important role in analysis of satellite images. As our need increases day by day but we are limited to natural resources on the earth and also we need to analyze the atmosphere above the earth surface. Therefore we need to check all these resources availability and this can be done by monitoring those things which are very important because it can provide valuable source of information for decision making process. Also in military application change detection can provide valuable information and help them in making decision process.

We have different existing methods which are used to detect changes under two categories supervised and unsupervised methods. In supervised method we need to perform training additionally in order to classify the information which means that we can predict our output by these classifier training. In unsupervised method we do not require any additional training to classify the information which means output is not predictable. In other words unsupervised method has ability to classify the information by itself so that it attracts more and more researcher to take interest.

Two techniques were introduced to solve unsupervised change detection problem which were expectation

Maximum (EM) base and Markov Random Field (MRF) based method. In the first method, selection of decision

threshold by which minimum change detection error was to be carried out. In the second method we analyze the difference image through pixel by pixel considering spatial information based on MRFs. These both techniques generally applicable to color image as they are not appropriate methods that can be applicable satellite images which have high resolution as compared to general color image. After change detection based on neural architecture when proposed then it becomes convenient approach to detect change on very high resolution satellite images and this method can deals with both multiband and multitemporal data for real change identification.

SAR (synthetic aperture Radar) data are those satellite images which are captured by this SAR device which is mounted on space aircraft, satellites and military aircrafts. The image captured by SAR is of very high resolution as compare to other satellite devices and are multispectral images and to process those we need to reduce the size of image without loss of necessary information. There are many methods such as K-means clustering, Multivariate Alteration Detection (MAD), and Change Vector Analysis (CVA), image differencing and other various methods. But some unsupervised methods are the best method by which we can easily distinguish between two images.

In earlier days we apply comparison or difference image based technique for low or medium resolution satellite images which employs the Bayesian theory to achieve the analysis of difference images. The technique such as image difference and image rationing are effective in detecting change in low dimension image as because they have to compare each image pixel by pixel. So they check all pixels and then result for changed map comes out. But they are not suitable for high resolution image because there numbers of pixels are of large size and computing all those pixels of images take more time and it also become complex to detect changed pixels. Therefore these techniques do not provide best result and also they are not efficient for such high resolution satellite images. But as unsupervised method of change detection came into existence then this method become effective in order to detect change of satellite images which are of good quality and or of good resolution.

In order to provide better change detection in SAR data set we apply unsupervised method which is appropriate to detect change in multitemporal SAR data set. The technique which is proposed in this letter comes under unsupervised method is Principal component Analysis (PCA). As PCA technique provide good and effective result in order to detect change.

\section{METHODOLOGY USED FOR CHANGE DETECTION}

Let us consider two registered multispectral images $\mathbf{I}_{\mathrm{t} 1}$ and $\mathbf{I}_{\mathrm{t} 2}$ captured by SAR of same geographical area and of same size (i.e. of same dimensions) taken at different time $\mathrm{t} 1$ and $\mathrm{t} 2$. And what we need to do is to detect the changes in the images by analyzing the images using the PCA technique. 
Principal Component Analysis (PCA) is a linear transformation of given data set into the new data set such that largest variance by any projection of data set lie on the first axis(also called first principal component), the second largest variance on the second axis, and further on. It is a technique by which it reduces the numbers of related variables associated with the given data set into the new set of variables which are uncorrelated but retain most of the variability that are associated with original variable of the data set.

Suppose that $\mathbf{x}$ is a vector of $m$ random variables, and that the variances of the $m$ random variables and the structure of the co variances or correlations between the $m$ variables are of interest. Unless $m$ is small, or the structure is very simple, it is not always very helpful to simply look at the $m$ variances and all of the $(1 / 2) \mathrm{m}(m-$ 1) correlations or co variances. An alternative approach is to look for a few $(<<m)$ derived variables that preserve most of the variability information.

The first step is to look for a linear function $z l=$ $\boldsymbol{\alpha} 1$ 'x of the elements of $\mathbf{x}$ having maximum variance, where $\mathbf{a}_{1}$ is a vector of $m$ constants $\mathrm{a}_{11}, \mathrm{a}_{12}, \mathrm{a}_{13}, \ldots \ldots, \mathrm{a}_{1 \mathrm{~m}}$ and ' denotes transpose, so that,

$$
\begin{gathered}
\mathbf{z}_{1}=\mathrm{a}_{1}{ }^{\prime} x=\mathrm{a}_{11} \mathrm{x}_{1}+\mathrm{a}_{22} \mathrm{x}_{2}+\mathrm{a}_{33} \mathrm{x}_{3}+\ldots \ldots .+\mathrm{a}_{1 \mathrm{~m}} \mathrm{x}_{\mathrm{n}} \\
\mathrm{a}_{1}=\arg \left\{\max \left(\operatorname{var}\left(\mathrm{z}_{1}\right)\right)\right\}
\end{gathered}
$$

The next step is to look for a linear function $a_{1}{ }^{\prime} x$ uncorrelated with $\mathrm{a}_{2}$ 'x having maximum variance, and so on, so that the kth stage a linear function of $\boldsymbol{\alpha}_{k}{ }^{\prime} \mathrm{x}$ is found that has maximum variance subject to being uncorrelated with $\mathrm{z}_{1}, \mathrm{z}_{2}, \mathrm{z}_{3}, \ldots \ldots, \mathrm{z}_{\mathrm{k}-1}$.

The kth derived variable, $a_{k}{ }^{\prime} x$ is the kth principal component. Up to $m$ principal components could be found, but in most applications the variability in $\mathbf{x}$ is accounted for by few, $\mathrm{n} \ll m$ principal components.

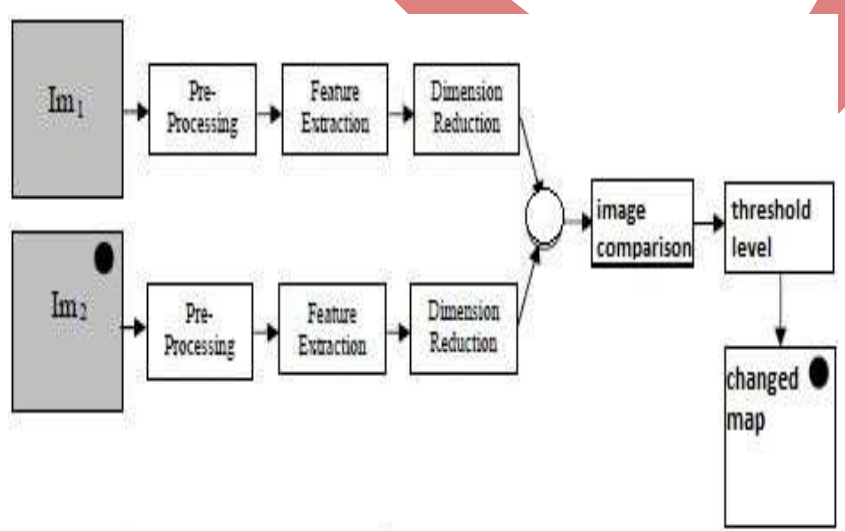

Figure 1: steps for change detection

Change detection using PCA involves following steps to be carried out in order to find those changes in SAR data taken at different time for same geographical area.

\section{Image Pre-processing}

In this step or process we compare the two images with respect to their sizes and also compare their spatial and spectral domain. As the size of both the images must be of same, any variation in size of image may lead to wrong output, so dimension of image must be same. In spatial domain we check both the images should co-register to that they associated with same area. Spatial domain contains the pixels which are associated with the particular area. And in spectral domain we check the change in illumination and atmospheric condition between the two acquisition times.

\section{Feature Extraction}

This step involves the extraction of new feature which is derived from original feature by means of some functional mapping keeping as much information as possible. Here using PCA technique this feature extraction can be done easily by generating principal component for the two images which are registered. Principal component are those which provide a new set of variable that are uncorrelated and are ordered so that it retain most of the variability present in the original variables. Here set of variables are the pixels associated with both of the two images which are taken and generating principal component for both the images and these principal component gives all information related to both the images. And principal component of image I $t 1$ is different from the principal component of image I $t 2$.

\section{Dimensionality Reduction}

One of the most important characteristics of PCA is it provides an effective dimensionality reduction in the feature and retain only those feature which have large variance to represent data. So here we reduce the size of image based on their principal component vector as we reduce the number of pixels associated with the images and we remain with those pixels which are helpful to give all information related to the images. And we perform this process separately for both the two images so that we can easily detect the change by reducing the number of comparison of pixels associated with two of the images.

\section{Image Comparison}

After finding the principal component for both the two images and reducing the dimension of the images we have now two different images and we are in state to compare both the images. Comparison of images is done through pixel by pixel and computes those pixels which are associated with the changed areas. Then we map that pixel in order to generate the change map.

\section{Threshold level}

This level is the most important part of change detection method as it involves the selection of threshold in a manner by which we can decide whether the pixel of particular image $\left(\mathbf{I}_{\mathrm{t} 1}\right)$ is change from one time to another taken at different interval $\left(\mathbf{I}_{\mathrm{t} 2}\right)$. In other way we check the number of principal components of 
image $\mathbf{I}_{\mathrm{t} 1}$ is different from the principal components of image $\mathbf{I}_{\mathrm{t} 2}$.

\section{EXPECTED RESULT}

In order to evaluate the performance of proposed method we will compare it with other traditional methods which are used in satellite images. The method proposed in this paper is an approach to get better result to detect change on SAR data. When we will compare with other traditional method such as SVM and Kmeans clustering technique we will find it will produce good result in an efficient manner. As in SVM we check through generated support vector for the images captured by SAR having same geographical region and compare them. This technique will detect the change but produces change map of less quality or less clarity.

In k-means clustering technique the data set related to images get clustered and classify that data set according to the information represented by the image We classify the data or information represented by images as water, land, forest, bridges, cities etc. When two images captured by SAR are compared with kmeans clustering technique, the images are checked by their clustering of their data and compare the clustered data of both the images to detect the change on SAR data. The result will show that clustering does not produce clear changed map and much noise contain by the changed map.

On applying PCA technique as proposed in this paper comparison of images done by comparing the Principal Component generated for both the images. PCA which reduces the size of image but also done feature selection by which it select those variable which are necessary for representing the information of image. On comparing the images captured by SA, it will compare those variables which are different from each other and detect the changed variables which are helpful in forming changed map. And this PCA technique will produce a changed map in an efficient manner and it will be clearer than any other techniques.

\section{CONCLUSION AND FUTURE WORK}

In this letter, PCA technique can provide best result for change detection in SAR data by processing the two different image of same geographical area and compare both them through pixel by pixel or through principal component vector generated for both image. The PCA technique easily classifies the changed area and the unchanged area by using principal component. This PCA technique not only detect the changes in the images but also convert a very high dimension image into lower one maintaining all information related to original image. So we can easily store as it occupy less storage space as compared to original one and it is an efficient technique.

In future PCA technique can be used in telemedical sciences in which it can be used for detecting diseases related to internal human body organ by comparing the multispectral images of human body organ of specified location.

\section{REFERENCES}

[1] F. Bovolo, "A multilevel parcel-based approach to change detection in very high resolution multitemporal images," IEEE Geosci. Remote Sens. Lett, vol. 6, no. 1, pp. 33-37, Jan. 2009.

[2] F. Pacifici, F. Del Frate, C. Solimini, and W. Emery, "An innovative neural net method to detect temporal changes in high-resolution optical satellite imagery," IEEE Trans. Geosci. Remote Sens., vol. 45, no. 9, pp. 2940-2952, Sep. 2007.

[3] F. Pacifici and F. Del Frate, "Automatic change detection in very high resolution images with pulsecoupled neural networks," IEEE Geosci. Remote Sens. Lett, vol. 7, no. 1, pp. 58-62, Jan. 2010.

[4] L. Bruzzone and D. Prieto, "Automatic analysis of the difference image for unsupervised change detection," IEEE Trans. Geosci. Remote Sens., vol. 38, no. 3, pp. 1171-1182, May 2000.

[5] C. Benedek and T. Sziranyi, "Change detection in optical aerial images by a multilayer conditional mixed Markov model," IEEE Trans. Geosci. Remote Sens., vol. 47, no. 10, pp. 3416-3430, Oct. 2009.

[6] T. Celik, "Change detection in Satellite images using a genetic algorithm approach," IEEE Geosci. Remote Sens. Lett, vol. 7, no. 2, pp. 386-390, Apr. 2010.

[7] T. Celik, "Unsupervised change detection in satellite image using principal component analysis and $k$-means clustering," IEEE Geosci. Remote Sens.Lett, vol. 6, no. 1, pp. 33-37, Jan. 2009.

[8] C. Yung-Yu, B. Curless, D. H. Salesin, and R. Szeliski, "A Bayesian approach to digital matting," in $C V P R, 2001$. 